\title{
Curcumin suppresses AGEs induced apoptosis in tubular epithelial cells via protective autophagy
}

\author{
YING WEI ${ }^{1-3}$, JIAQI GAO ${ }^{1-3}$, LINGLING QIN $^{2,3}$, YUNLING XU ${ }^{1-3}$, HAOXIA SHI $^{1-3}$, \\ LINGXIA QU ${ }^{1-3}$, YONGQIAO LIU ${ }^{1-3}$, TUNHAI XU ${ }^{1-3}$ and TONGHUA LIU ${ }^{2,3}$ \\ ${ }^{1}$ School of Chinese Pharmacy, Beijing University of Chinese Medicine, Beijing 100102; \\ ${ }^{2}$ Health Cultivation Key Laboratory of The Ministry of Education; ${ }^{3}$ Health Cultivation Key Laboratory of \\ Beijing, Beijing University of Chinese Medicine, Beijing 100029, P.R. China
}

Received June 11, 2017; Accepted October 5, 2017

DOI: $10.3892 / \mathrm{etm} .2017 .5314$

\begin{abstract}
Renal tubular cell apoptosis and tubular dysfunction is an important process underlying diabetic nephropathy (DN). Understanding the mechanisms underlying renal tubular epithelial cell survival is important for the prevention of kidney damage associated with glucotoxicity. Curcumin has been demonstrated to possess potent anti-apoptotic properties. However, the roles of curcumin in renal epithelial cells are yet to be defined. The present study investigated advanced glycation or glycoxidation end-product (AGE)-induced toxicity in renal tubular epithelial cells via several complementary assays, including cell viability, cell apoptosis and cell autophagy in the NRK-52E rat kidney tubular epithelial cell line. The extent of apoptosis was significantly increased in the NRK-52E cells following treatment with AGEs. The results also indicated that curcumin reversed this effect by promoting autophagy through the phosphoinositide 3-kinase/AKT serine/threonine kinase signaling pathway. These conclusions suggested that curcumin exerts a renoprotective effect in the presence of AGEs, at least in part by activating autophagy in NRK-52E cells. Collectively, these findings indicate that curcumin not only exerts renoprotective effects, however may
\end{abstract}

Correspondence to: Professor Tunhai Xu, School of Chinese Pharmacy, Beijing University of Chinese Medicine, 6 Wangjing Middle Ring South Road, Beijing 100102, P.R. China

E-mail: th2015_11@sina.com

Abbreviations: NRK-52E cells, normal rat kidney tubular epithelial cells; EMT, epithelial-to-mesenchymal transition; MTT, 3-[4,5-dimethylthiazol-2-y]-2,5-diphenyltetrazolium bromide; DMEM, Dulbecco's modified Eagle's medium; DMSO, dimethyl sulfoxide; BSA, bovine serum albumin; PBS, phosphate-buffered saline; TBS, Tris-buffered saline; DN, diabetic nephropathy; AGEs, advanced glycation or glycoxidation end-products; DM, diabetes mellitus; 3-MA, 3-methyladenine; FBS, fetal bovine serum; TUNEL, the terminal uridine nick 3 ' end labeling

Key words: apoptosis, autophagy, AGEs, curcumin, diabetic nephropathy also act as a novel therapeutic strategy for the treatment of diabetic nephropathy.

\section{Introduction}

Diabetic nephropathy (DN) previously known as idiopathic nodular glomerulosclerosis is the leading cause of end-stage renal disease. It is characterized by increased blood pressure, increased urinary albumin and glomerular lesions, leading to the loss of glomerular filtration is the main microvascular complications of diabetes (DM) $(1,2)$. There are a variety of mechanisms believed to be involved, including non-enzymatic glycosylation, polyol pathway theory and protein kinase $C$ theory (3-5). What is the most widely accepted hypothesis of DN is the non-enzymatic glycoside theory. In the long-term hyperglycemic state, glucose molecules and protein in vivo occur non-enzymatic glycosylation reaction and yield advanced glycation end products (advanced glycation or glycoxidation end-products, AGEs), leading to the occurrence of the disease $(6,7)$. Lots of studies have confirmed that eliminating the effects of AGEs can reduce renal damage, inhibit the progress of complications of diabetes and promote the regeneration of renal cells, but there are few studies focus on these $(8,9)$. Some potential targets for renoprotective therapies, including AGEs inhibitors, PKC inhibitors, have been studied and tested in cells and animals models, the development of diabetic nephropathy remains a major concern. Therefore, more specific pathophysiologic mechanisms underlying DN should be explored and identified to develop new treatment and strategies for this devastating disease.

Previous study found that AGEs could induce apoptosis and dysfunction of tubular cells may contribute in part to glomerular hyperfiltration, an early renal dysfunction in diabetes, so that late glomerulosclerosis $(10,11)$. Autophagy engages in complex interplay with apoptosis. Apoptosis and autophagy occur simultaneously within the same cell under the stimulation of various stress including starvation, hypoxia and ER stress (12). In the present study, we explored the relationship between autophagy and apoptosis under AGEs treatment. Whether curcumin could reduce AGEs-induced cell apoptosis and what role of autophagy in this process. 
Furthermore, we determined whether curcumin exerts a protective effect through autophagy pathway in response to AGEs.

\section{Materials and methods}

Materials. Curcumi, AGEs, Triton X-100, DMSO, LY294002 and 3-methyladenine (3-MA) were purchased from Sigma-Aldrich (Merck KGaA, Darmstadt, Germany). DMEM and fetal bovine serum (FBS) were both obtained from HyClone (Thermo Fisher Scientific, Inc., Logan, UT, USA). Anti-GAPDH, anti-bax, anti-AIF, anti-caspase-3 and anti-p-AKT were obtained (Santa Cruz Biotechnology, Inc., Dallas, TX, USA). Anti-Beclin 1 and anti-LC3 were both obtained from Cell Signaling Technology, Inc. (Beverly, MA, USA). ECL kit was purchased (Pierce; Thermo Fisher Scientific, Inc.). Flow cytometer (FACSCalibur; Becton-Dickinson, Franklin Lakes, NJ, USA). All reagents used were trace element analysis grade. All water used was glass distilled.

Cell culture. Rat kidney tubular epithelial cell line NRK-52E was purchased from the American Type Culture Collection (ATCC; Manassas, VA, USA). The cells were cultured in a $5 \% \mathrm{CO}_{2}$ atmosphere in Dulbecco's modified Eagle's medium (DMEM; low glucose), supplemented with $10 \%$ fetal bovine serum, $4 \mathrm{mM} \mathrm{L-glutamine} \mathrm{and} 1 \%$ penicillin/streptomycin at a density of $6 \times 10^{3}$ cells/well in six-well culture plates.

Cell viability. We use 3-(4,5-dimethylthiazol-2-yl)-2,5diphenyltetrazolium bromide (MTT) assay to detect cell viability. Briefly, the sample medium were added to $10 \mu \mathrm{l}$ MTT $(500 \mu \mathrm{g} / \mathrm{ml})$ and incubated for $3 \mathrm{~h}$ at $37^{\circ} \mathrm{C}$ following treatment. Subsequently, the MTT solution was removed and $100 \mu \mathrm{l}$ dimethyl sulfoxide (DMSO) was added to dissolve the colored formazan crystals. The absorbance of each aliquot at $490 \mathrm{~nm}$ was measured using a Sunrise microplate reader (Tecan Group Ltd., Männedorf, Switzerland). The cell viability was determined as the ratio of the signal.

Measurement of apoptosis by flow aytometry and terminal uridine nick 3' end labelling (TUNEL) assays. We detected apoptosis by Annexin V labeled with FITC. Propidium iodide was used to determine cell necrosis. After exposure to various experimental conditions, cells were trypsinized and labeled with fluorochromes at $37^{\circ} \mathrm{C}$, and then cytofluorometric analysis was performed with a FACS can (Becton-Dickinson).

Apoptosis was also evaluated by the TUNEL method. For the TUNEL assays, cells grown on a coverslip were pretreated with various experimental conditions. The TUNEL assay kit was used to detect apoptotic cells under a fluorescence microscope (Leica TCS SPE; Leica Microsystems GmbH, Wetzlar, Germany). After treatment, the cells were washed with PBS, fixed in $4 \%$ paraformaldehyde/PBS, and permeabilized with $0.2 \%$ Triton X-100 in citrate buffer. Samples were incubated with TdT and fluorescein-labeled dUTP, counterstained with 4',6-diamidino-2-phenylindole (DAPI), and then observed under a fluorescence microscope (Leica TCS SPE). Percentages of apoptotic cells were estimated by counting 300 cells in random fields.
Immunofluorescence staining. NRK-52E cells were fixed with $4 \%$ paraformaldehyde at $4^{\circ} \mathrm{C}$ for $30 \mathrm{~min}$, incubated with $0.2 \%$ Triton X-100 for $10 \mathrm{~min}$. The cells were blocked at non-specific antibody binding sites by incubating with $10 \%$ goat serum in PBS containing $0.3 \%$ Triton $\mathrm{X}-100$ and $0.5 \%$ bovine serum albumin (BSA) for $30 \mathrm{~min}$ at room temperature, followed by incubation with a mouse monoclonal antibody against Beclin 1 and LC3 (1:400 in PBS; Cell Signaling Technology, Inc.) overnight. Then TRITC and FITC-conjugated goat anti-mouse IgG (1:100 in PBS) was used to incubate for $0.5 \mathrm{~h}$ at room temperature. Heochst 33342 was added to the cells for $15 \mathrm{~min}$. After washing three times with PBS, cells were visualized under fluorescence microscopy.

Western blot analysis. The NRK-52E cells were lysed in protein extraction reagent (Tissue Protein Extraction kit; Pierce, Rockford, IL, USA) and the lysates were extracted by centrifugation. Equal amounts of protein were loaded per sample in each experiment, separated by SDS-PAGE and transferred to polyvinylidene difluoride membranes by electroblotting. The protein-bound membranes were blocked and washed in Tris-buffered saline (TBS)-Tween-20. The membranes were incubated overnight with primary antibodies. The primary antibodies used in the present study were as follows: anti-GAPDH, anti-bax, anti-AIF, anti-caspase-3 and anti-p-AKT (Santa Cruz Biotechnology, Inc.). Anti-Beclin 1 and anti-LC3 (Cell Signaling Technology, Inc.). After washing in TBS-0.1\% Tween-20, the membranes were then incubated with horseradish peroxidase-conjugated secondary antibodies overnight at $4^{\circ} \mathrm{C}$. Finally, the blots were developed using an enhanced chemiluminescence system (Pierce). To compare the levels of proteins, the density of each signal was evaluated by image analysis software (CS Analyzer; ATTO, Tokyo, Japan).

Statistical analysis. Statistical comparison was made on the differences in mean values among multiple groups by using one-way analysis of variance with post hoc Scheffe's test. Statistical significance was achieved if P-values were $<0.05$.

\section{Results}

AGEs inhibits cell viability in NRK-52E cells. The cell viability of NRK-52E cells under AGEs conditions were assessed by MTT. As shown in (Fig. 1A), when the cells were treated with 0-2,000 $\mu \mathrm{g} / \mathrm{ml}$ AGEs for 24, 48 and $72 \mathrm{~h}$, AGEs significantly inhibited NRK-52E cells viability in concentration-dependent manner. Similarly, when the cells were treated with 300, 700 and 1,000 $\mu \mathrm{g} / \mathrm{ml}$ AGEs for 0-72 h, AGEs inhibited NRK-52E cell viability in time-dependent manner (Fig. 1B).

Curcumin inhibits AGEs-induced cell apoptosis. Curcumin, the active ingredient from the spice turmeric (Curcuma longa L.), has been demonstrated recently to possess anti-apoptosis effects (13). This study aimed to investigate the protective effect of curcumin on AGEs induced apoptosis and the underlying mechanism in NRK-52E cells. We then further hypothesize that curcumin may also play a role in the regulation of apoptosis under AGEs. As shown in (Fig. 2), the NRK-52E cells were treated with $700 \mu \mathrm{g} / \mathrm{ml}$ AGEs with or without $10 \mu \mathrm{M}$ curcumin for $48 \mathrm{~h}$. Firstly, AGEs-induced apoptosis 

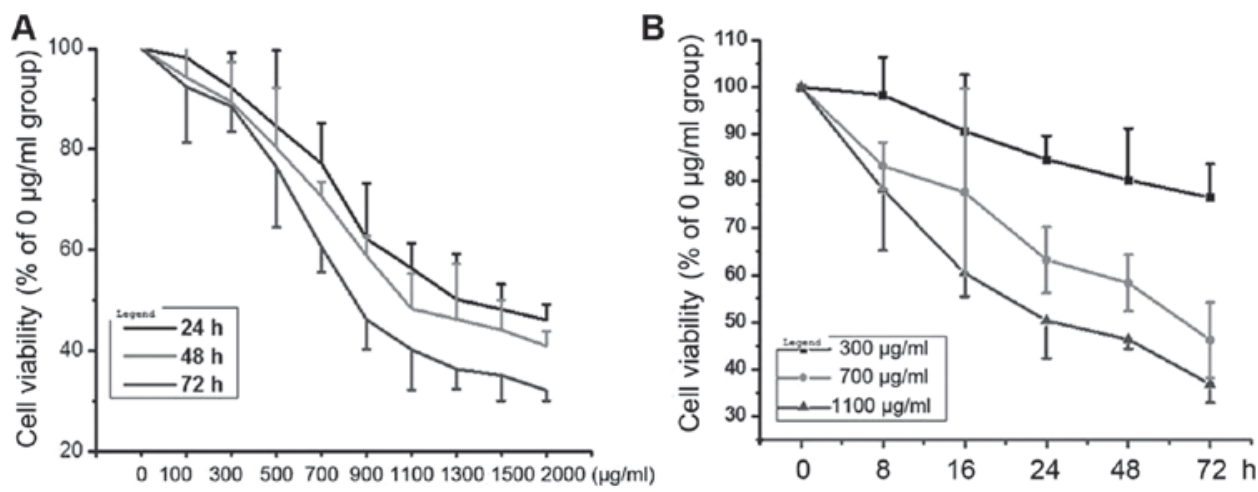

Figure 1. AGEs decreased the cell viability in NRK-52E cells. (A) Cells were treated with $0-2,000 \mu \mathrm{g} / \mathrm{ml}$ AGEs for 24,48 and $72 \mathrm{~h}$, and the cell viability were detected by MTT. (B) Cells were treated with 300, 700 and 1,000 $\mu \mathrm{g} / \mathrm{ml}$ AGEs for 0-72 h, and the cell viability were detected by MTT.

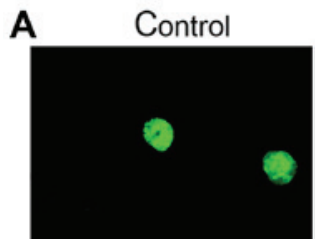

Curcumin
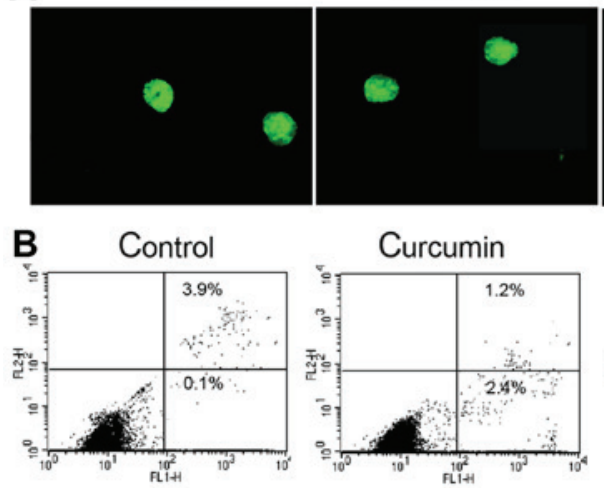

C

Bax

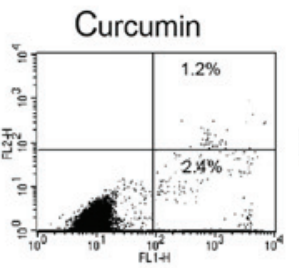

AGES

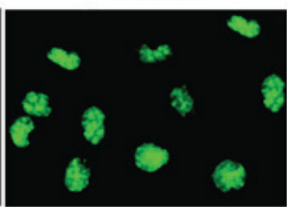

AGES

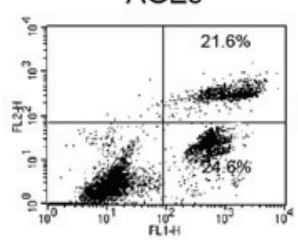

AGEs+curcumin

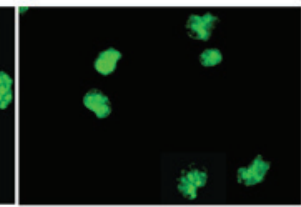

AGEs+curcumin

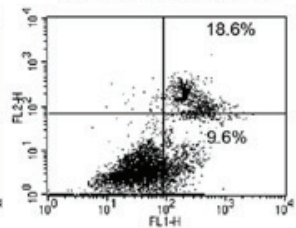

AIF
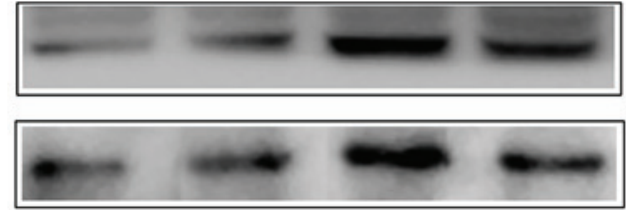

pro-caspase-3

Cleaved caspase- 3

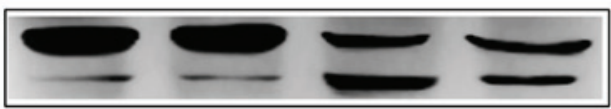

GAPDH

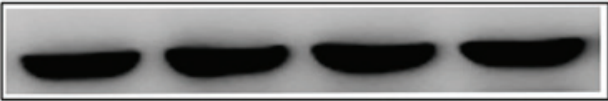

AGEs

Curcumin
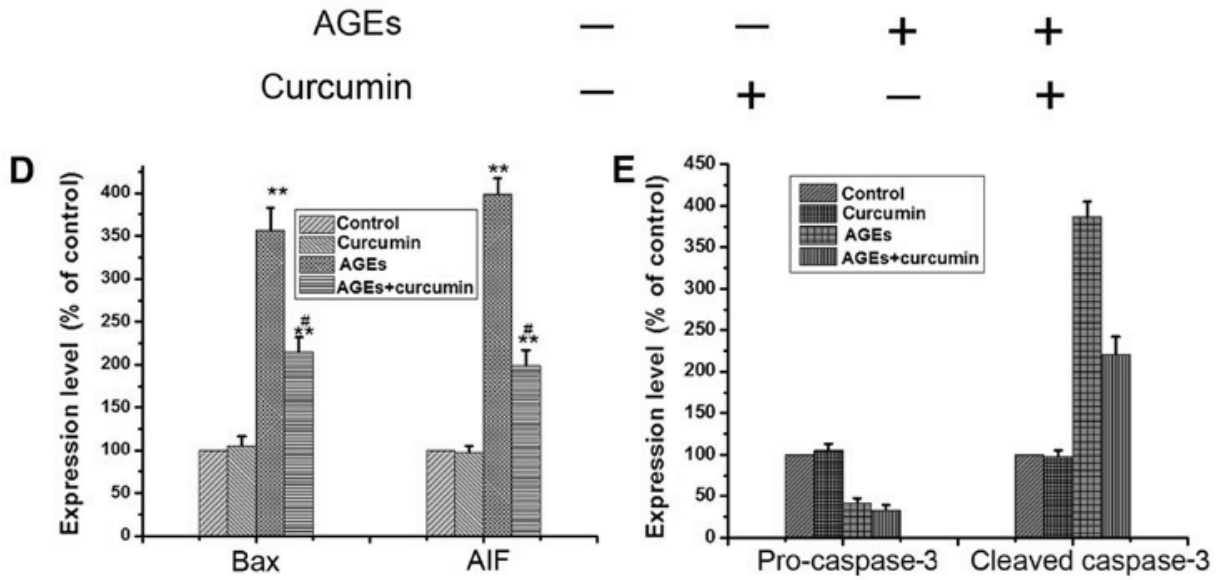

Figure 2. The effect of curcumin on cell apoptosis induced by AGEs. (A) Cells were treated with $700 \mu \mathrm{g} / \mathrm{ml}$ AGEs with or without $10 \mu \mathrm{M}$ curcumin for $48 \mathrm{~h}$, and the apoptosis was determined by TUNEL analysis. (B) Cells were treated with above, and the apoptosis was determined by flow cytometry followed by Annexin V/PI double staining. Each value represented mean \pm SEM $(n=6)$. (C-E) Cells were treated with above, and the expression of bax, AIF and caspase-3 were detected by western blot analysis. Corresponding protein levels were assessed using densitometry and are expressed as relative intensities. GAPDH was used as loading control. Each value represents the mean $\pm \mathrm{SEM}(\mathrm{n}=6)\left({ }^{* *} \mathrm{P}<0.01\right.$ vs. control; ${ }^{*} \mathrm{P}<0.01$ vs. AGEs group). 
A

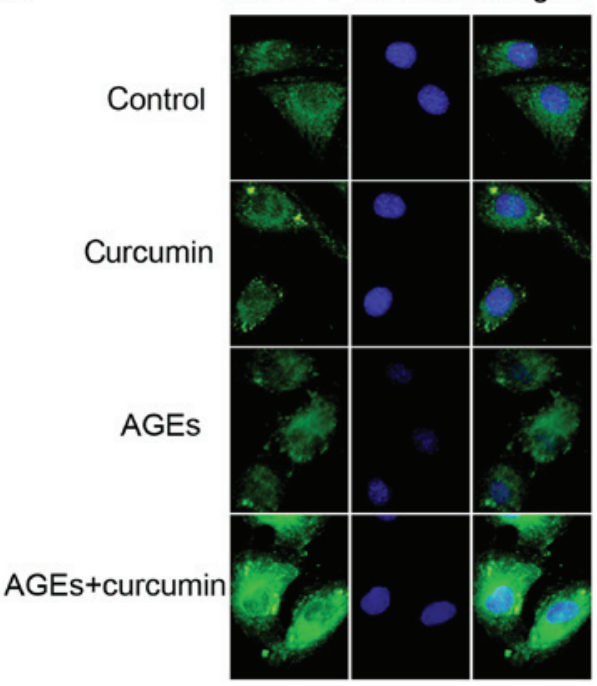

C

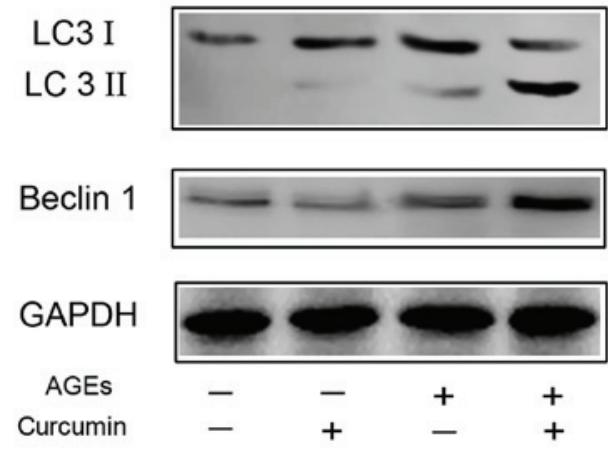

B
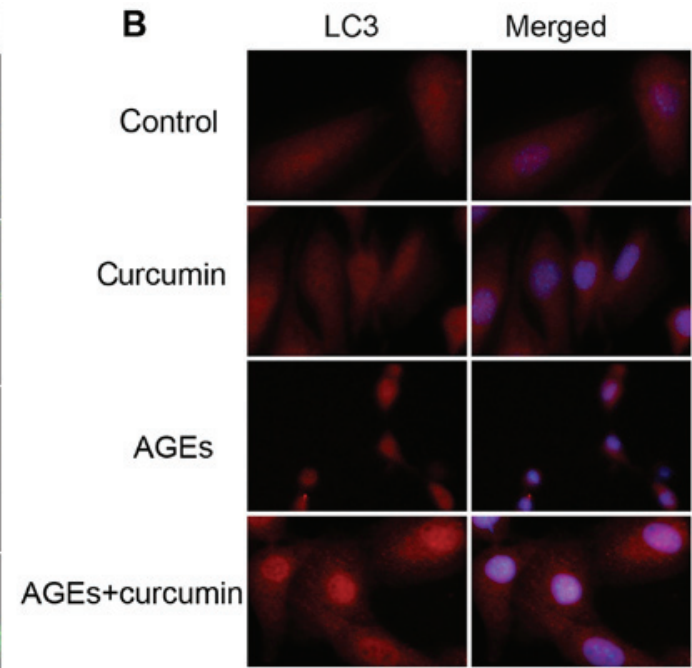

D

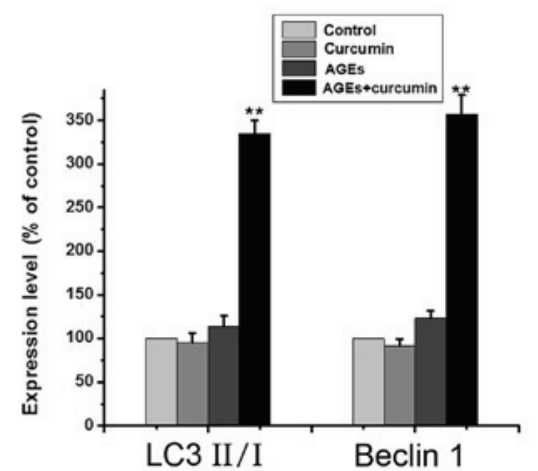

Figure 3. Curcumin promoted autophagy in NRK-52E cells. (A) Cells were treated with $700 \mu \mathrm{g} / \mathrm{ml}$ AGEs with or without $10 \mu \mathrm{M}$ curcumin for $48 \mathrm{~h}$, and the Beclin 1 protein was stained and observed under a fluorescence microscope as described in Materials and methods. (B) Cells were treated with above, and the LC3 protein was stained and observed under a fluorescence microscope as described in Materials and methods. (C and D) Cells were treated with above, and the expression of LC3 and Beclin 1 were detected by western blot analysis. The results were representatives of three independent experiments. GAPDH was used as loading control ( ${ }^{* *} \mathrm{P}<0.01$ vs. control).

was determined by TUNEL analysis. As can be seen from (Fig. 2A), AGEs treatment alone led to a significant increase in the apoptotic population, while curcumin cotreatment resulted in a substantial decrease of AGEs-induced NRK-52E cells apoptosis. We further evaluated the anti-apoptotic effect of curcumin by directly assessing the percentage of apoptotic cells using flow cytometry followed by Annexin V/PI double staining. Addition of curcumin reduced the percentage of AGEs-induced apoptotic cells from 46.2 to $28.2 \%$ (Fig. 2B). To further confirm the anti-apoptotic effect of curcumin, we performed western blot analysis. AGEs injury is associated with upregulation of Bax and AIF, leading to caspase cascade activation (caspase-3), However, co-incubation of NRK-52E cells with curcumin significantly reduced AGEs-induced apoptosis-related proteins (Fig. 2C-E). These results indicate that curcumin activates cytoprotective effect in AGEs induced apoptosis.

Curcumin enhances autophagy in NRK-52E cells. Apoptosis and autophagy are two evolutionarily conserved processes that maintain homeostasis during stress. The crosstalk between apoptosis and autophagy is complex, as autophagy can function to promote cell survival under various cellular conditions. In this text, we explored the role of autophagy in the cytoprotective effect of curcumin. As above, the NRK-52E cells were treated with $700 \mu \mathrm{g} / \mathrm{ml}$ AGEs with or without $10 \mu \mathrm{M}$ curcumin for $48 \mathrm{~h}$. After both curcumin and AGEs treatment, the number of LC3 and Beclin 1 fluorescent dots dramatically increased (Fig. 3A and B), suggesting that autophagic vacuolization occurs in response to curcumin treatment, compared to the AGEs alone group, From the results of fluorescence microscope. However, treatment of curcumin alone did not increase autophagy in NRK-52E cells (Fig. 3A and B). Similar changes were observed on western blot results. The expressions of Beclin 1 and the ratio of LC3II and LC3I were significantly increased in AGEs + curcumin group compared to AGEs group $(\mathrm{P}<0.01)$ (Fig. 3C and D). Theses results indicate that curcumin promotes autophagy in NRK-52E cells.

Curcumin inhibits apoptosis through protective autophagy. To investigate whether the mechanism of curcumin anti-apoptotic effects was the induction of chondrocyte autophagy, 3-Methyladenine (3-MA), a type of potent pharmacological inhibitors on autophagy, was used to suppress the autophagy. As (Fig. 4A) demonstrated that pretreatment with 2 mM 3-MA could block autophagy in NRK-52E cells without significant cytotoxicity. From the results of (Fig. 4A), curcumin significantly inhibited AGEs-induced apoptosis, 

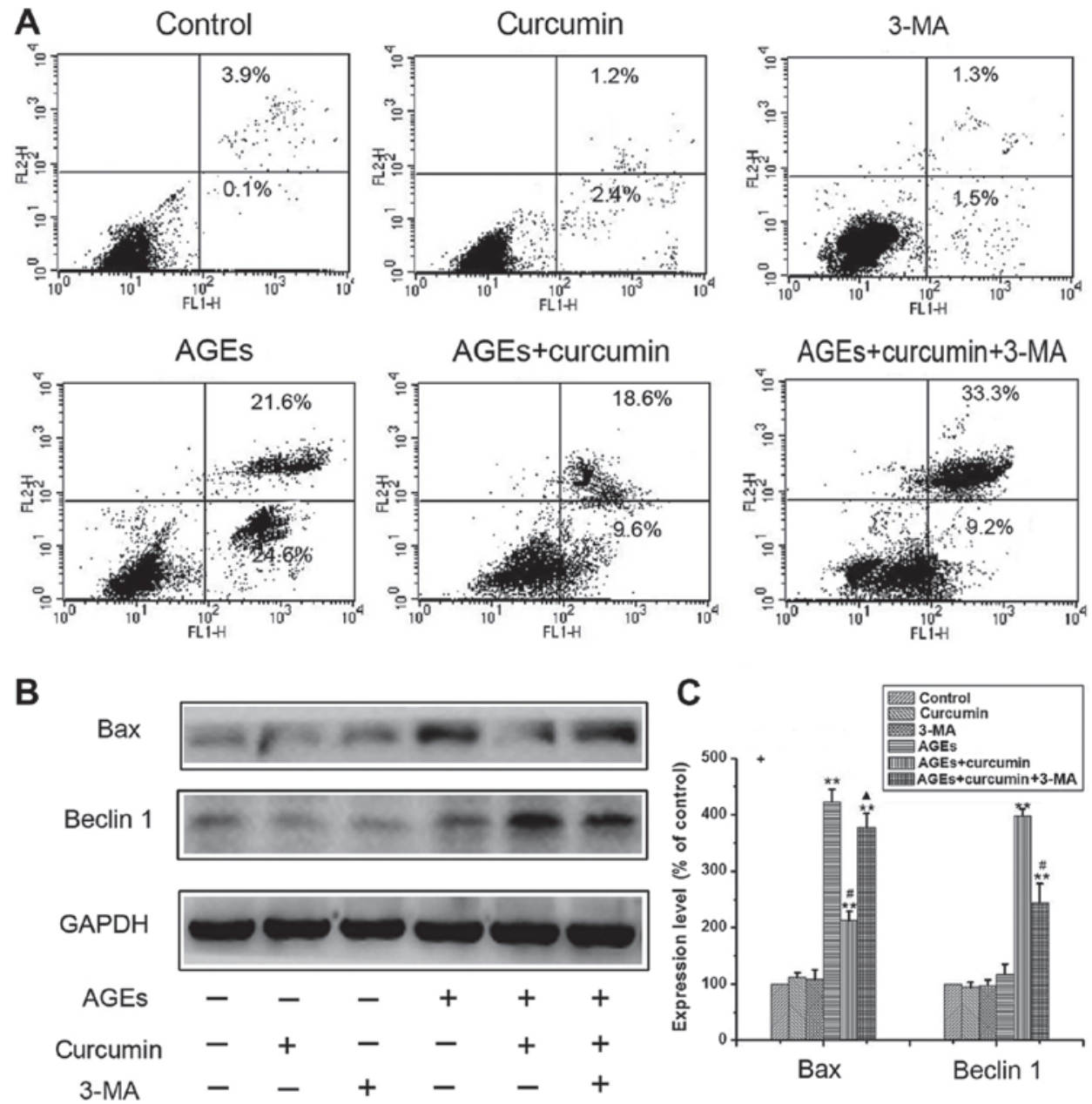

Figure 4. The role of autophagy in the protective effect of curcumin. (A) Cells were treated with $700 \mu \mathrm{g} / \mathrm{ml}$ AGEs, $10 \mu \mathrm{M}$ curcumin, as well as 2 mM 3-MA for $48 \mathrm{~h}$ or a combined treatment of $700 \mu \mathrm{g} / \mathrm{ml}$ AGEs and $10 \mu \mathrm{M}$ curcumin or $2 \mathrm{mM} 3-\mathrm{MA}, 700 \mu \mathrm{g} / \mathrm{ml}$ AGEs and $10 \mu \mathrm{M}$ curcumin for $48 \mathrm{~h}$, and apoptosis was determined by flow cytometry followed by Annexin V-PI double staining. (B and C) Cells were treated aboved, and the expression of Bax and Beclin 1 were detected by western blot analysis. The results were representatives of three independent experiments. GAPDH was used as loading control $\left({ }^{* *} \mathrm{P}<0.01\right.$ vs. control; ${ }^{\prime} \mathrm{P}<0.05$ vs. AGEs group; ${ }^{\mathbf{A}} \mathrm{P}>0.05$ vs. AGEs group).
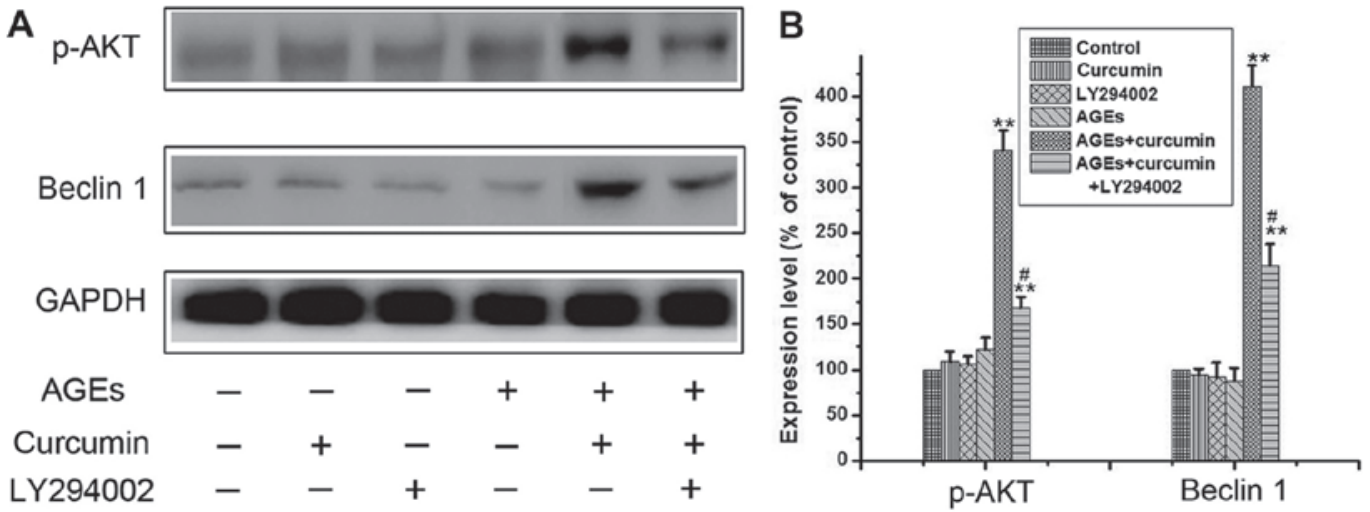

Figure 5. The role of PI3K/AKT signaling pathway in authphagy. (A) Cells were treated with $700 \mu \mathrm{g} / \mathrm{ml}$ AGEs, $10 \mu \mathrm{M}$ curcumin, as well as $10 \mathrm{mM}$ LY294002

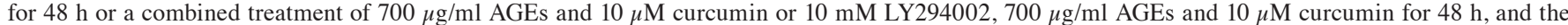
expression of p-AKT, Beclin 1 were determined by western blot analysis. (B) The results were representatives of three independent experiments. GAPDH was used as loading control ( ${ }^{* *} \mathrm{P}<0.01$ vs. control; ${ }^{\#} \mathrm{P}<0.05$ vs. AGEs group).

however, the protective role of curcumin was greatly reduced when co-cultured with autophagy inhibitor 3-MA. These results show that autophagy is an important pathway for a protective role of curcumin. In order to further validate this conclusion, we further carried out western blot experiment. The western blot analysis offered another evidence. The upregulation of the expression of apoptosic protein AIF, Bax and cleaved caspase- 3 were decreased when cells were 
co-treated with curcumin but this effect was attenuated by co-treatment with 3-MA (Fig. 4B and C). These results indicated that autophagy induced by curcumin serve in a protective manner, and blockage of autophagy interrupted the protection effect of curcumin in NRK-52E cells.

Curcumin upregulates autophagy through PI3k/AKT pathway. Given that PI3K/AKT pathway activation promotes autophagy in different cell lines (14). To investigate the involvement of PI3k/AKT pathway in curcumin-induced autophagy, $10 \mu \mathrm{M}$ LY294002 (the inhibitor of PI3K/AKT) was added to the medium of NRK-52E cells. As the results of Fig. 5A and B, preincubation of the cells with both curcumin and AGEs activated the PI3k/AKT signaling pathway and significantly increased the expression of Beclin 1 . However, the expression of Beclin 1 was greatly reduced when co-cultured with PI3k/AKT inhibitor LY294002. These results show that curcumin promotes autophagy through PI3K/AKT signaling pathway.

\section{Discussion}

Curcumin as a kind of plant polyphenol extracted from turmeric is the most important active ingredient of turmeric to play the pharmacological effect (15). Curcumin exhibits a variety of biological activities such as anti-inflammatory, antioxidation, anti-human immunodeficiency virus, anti-fibrosis and anticancer effects (16-18). During recent years, the defensive role of curcumin against apoptosis has been experimentally documented (19). To investigate whether curcumin could protect rat's kidney against diabetes damage, we assessed NRK-52E cells for apoptosis, autophagy and apoptotic factor activities. Firstly, our study confirmed that curcumin inhibited AGE-induced apoptosis and downregulated the expression of Bax, AIF and caspase-3. This results obtained from this study suggested that curcumin could reduce cytotoxic response of AGEs. Furthermore, we observed that the NRK-52E cells were treated with curcumin showed a significant activation of protective autophagy and upregulated the level of Beclin 1 and the ratio of LC3II and LC3I. Previous study found that curcumin could protect cells against oxidative stress-induced damage through inhibiting apoptosis and inducing autophagy (20). Our findings also confirm this conclusion. However, the role of autophagy in this process has not been reported.

The relationship between autophagy and apoptosis is complex and can be induced by both processes in response to similar stimuli, through which superfluous, damaged, or aged cells or organelles are eliminated (21). Autophagy as a double-edged sword is evolutionarily conserved mechanism for maintaining cellular homeostasis and plays a positive and negative role in promoting apoptosis $(22,23)$. Previous study highlighted that autophagy played protective roles in the prevention of diabetes through their ability to provide an adaptive response to insulin resistant states (24). Moreover, study also revealed that curcumin caused some novel cellular mechanisms to promote autophagy as a protective effect (25). Our findings also confirm this conclusion that curcumin both greatly attenuates AGE-induced apoptosis by activating autophagy and increases autophagy by activation of PI3K/AKT signaling pathway (a classical autophagic pathway).
In conclusion, we have shown the protective potential of curcumin for AGEs-mediated apoptosis. Autophagy caused by the activation of PI3K/AKT pathway plays a significant role in this process. Although the present study is limited to in vitro studies, it suggests that curcumin may control autophagy and play a protective role to resistance to diabetic kidney damage. The results also suggest that the controlling autophagy may have the therapeutic potential to inhibit cell apoptosis and prevent diabetic nephropathy.

\section{Acknowledgements}

This study was supported by The International Science and Technology Cooperation Program of China (2010DFB33260; 2010DFB33260).

\section{References}

1. Yamamoto Y, Kato I, Doi T, Yonekura H, Ohashi S, Takeuchi M, Watanabe T, Yamagishi S, Sakurai S, Takasawa S, et al: Development and prevention of advanced diabetic nephropathy in RAGE-overexpressing mice. J Clin Invest 108: 261-268, 2001.

2. Elsherbiny NM and Al-Gayyar MM: The role of IL-18 in type 1 diabetic nephropathy: The problem and future treatment. Cytokine 81: 15-22, 2016.

3. Kalia K, Sharma S and Mistry K: Non-enzymatic glycosylation of immunoglobulins in diabetic nephropathy. Clin Chim Acta 347: 169-176, 2004.

4. Hashimoto Y, Yamagishi S, Mizukami H, Yabe-Nishimura C, Lim SW, Kwon HM and Yagihashi S: Polyol pathway and diabetic nephropathy revisited: Early tubular cell changes and glomerulopathy in diabetic mice overexpressing human aldose reductase. J Diabetes Investig 2: 111-122, 2011.

5. Teng B, Duong M, Tossidou I, Yu X and Schiffer M: Role of protein kinase $\mathrm{C}$ in podocytes and development of glomerular damage in diabetic nephropathy. Front Endocrinol (Lausanne) 5: 179, 2014.

6. Fukami K, Yamagishi S, Ueda S and Okuda S: Role of AGEs in diabetic nephropathy. Curr Pharm Des 14: 946-952, 2008.

7. Kumar Pasupulati A, Chitra PS and Reddy GB: Advanced glycation end products mediated cellular and molecular events in the pathology of diabetic nephropathy. Biomol Concepts 7: 293-309, 2016.

8. Kishore L, Kaur N and Singh R: Renoprotective effect of Bacopa monnieri via inhibition of advanced glycation end products and oxidative stress in STZ-nicotinamide-induced diabetic nephropathy. Ren Fail 38: 1528-1544, 2016.

9. Chen C, Huang K, Hao J, Huang J, Yang Z, Xiong F, Liu P and Huang $\mathrm{H}$ : Polydatin attenuates AGEs-induced upregulation of fibronectin and ICAM-1 in rat glomerular mesangial cells and db/db diabetic mice kidneys by inhibiting the activation of the SphK1-S1P signaling pathway. Mol Cell Endocrinol 427: 45-56, 2016.

10. Maeda S,Matsui T, Takeuchi M and Yamagishi S: Sodium-glucose cotransporter 2-mediated oxidative stress augments advanced glycation end products-induced tubular cell apoptosis. Diabetes Metab Res Rev 29: 406-412, 2013.

11. Qi W, Niu J, Qin Q, Qiao Z and Gu Y: Glycated albumin triggers fibrosis and apoptosis via an NADPH oxidase/Nox4-MAPK pathway-dependent mechanism in renal proximal tubular cells. Mol Cell Endocrinol 405: 74-83, 2015.

12. Masini M, Bugliani M, Lupi R, del Guerra S, Boggi U, Filipponi F, Marselli L, Masiello P and Marchetti P: Autophagy in human type 2 diabetes pancreatic beta cells. Diabetologia 52: 1083-1086, 2009.

13. Jaroonwitchawan T, Chaicharoenaudomrung N, Namkaew J and Noisa P: Curcumin attenuates paraquat-induced cell death in human neuroblastoma cells through modulating oxidative stress and autophagy. Neurosci Lett 636: 40-47, 2017.

14. Zeng Y, Yang X, Wang J, Fan J, Kong Q and Yu X: Aristolochic acid I induced autophagy extenuates cell apoptosis via ERK 1/2 pathway in renal tubular epithelial cells. PLoS One 7: e30312, 2012.

15. Araújo CC and Leon LL: Biological activities of Curcuma longa L. Mem Inst Oswaldo Cruz 96: 723-728, 2001.

16. Menon VP and Sudheer AR: Antioxidant and anti-inflammatory properties of curcumin. Adv Exp Med Biol 595: 105-125, 2007. 
17. Liu PY: Curcumin: Another potential translational candidate for anti-fibrosis on heart failure? Acta Cardiol Sin 30: 483-484, 2014.

18. Sufi SA, Adigopula LN, Syed SB, Mukherjee V, Coumar MS, Rao HS and Rajagopalan R: In-silico and in-vitro anti-cancer potential of a curcumin analogue (1E, 6E)-1, 7-di (1H-indol-3-yl) hepta-1, 6-diene-3, 5-dione. Biomed Pharmacother 85: 389-398, 2017.

19. Guo S, Long M, Li X, Zhu S, Zhang M and Yang Z: Curcumin activates autophagy and attenuates oxidative damage in EA.hy926 cells via the Akt/mTOR pathway. Mol Med Rep 13: 2187-2193, 2016

20. Delou JM, Biasoli D and Borges HL: The complex link between apoptosis and autophagy: A promising new role for RB. An Acad Bras Cienc 88: 2257-2275, 2016.

21. Mizushima N: Autophagy and apoptosis. Nihon Rinsho Meneki Gakkai Kaishi 23: 527-530, 2000 (In Japanese).
22. Nie C, Zhou J, Qin X, Shi X, Zeng Q, Liu J, Yan S and Zhang L: Reduction of apoptosis by proanthocyanidin-induced autophagy in the human gastric cancer cell line MGC-803. Oncol Rep 35: 649-658, 2016.

23. Kim KY, Kim SH, Yu SN, Park SG, Kim YW, Nam HW, An HH, Yu HS, Kim YW, Ji JH, et al: Lasalocid induces cytotoxic apoptosis and cytoprotective autophagy through reactive oxygen species in human prostate cancer PC-3 cells. Biomed Pharmacother 88: 1016-1024, 2017.

24. Fujitani Y, Kawamori R and Watada H: The role of autophagy in pancreatic beta-cell and diabetes. Autophagy 5: 280-282, 2009.

25. Han J, Pan XY, Xu Y, Xiao Y, An Y, Tie L, Pan Y and Li XJ: Curcumin induces autophagy to protect vascular endothelial cell survival from oxidative stress damage. Autophagy 8: 812-825, 2012. 